\title{
Perspectiva de género en la violencia filio-parental ${ }^{*}$
}

\author{
Cómo citar este artículo: \\ Correa, S. M., Botero, Y., Valoyes, J. V. \\ y Rodríguez, A. (2021). Perspectiva de \\ género en la violencia filio-parental. Revista \\ Latinoamericana de Estudios de Familia, \\ 13(2), 143-162. https://doi.org/10.17151/ \\ rlef.2021.13.2.8
}

\author{
Sara Melisa Correa-Agudelo** \\ Yesica Botero-Arango*** \\ Jenifer Vanesa Valoyes-Arenas**** \\ Alexánder Rodríguez-Bustamante ${ }^{* * * * *}$
}

\begin{abstract}
Resumen: La violencia filio-parental es una manifestación de la violencia intrafamiliar que se caracteriza por la acción violenta, intencionada y sistemática de hijos hacia los progenitores o quien ejerce el rol de adulto significativo; el daño ocasionado a las víctimas puede comprender dimensiones físicas, psicológicas, emocionales y financieras. Objetivo. Reconocer las cualidades de la víctima y victimario de la violencia filio-parental en las dinámicas familiares desde una perspectiva de género. Metodología.

Esta investigación se realizó bajo un enfoque cualitativo con una metodología hermenéutica en la modalidad de estado del arte; se tuvieron en cuenta 56 referencias bibliográficas privilegiando los artículos de revista con un margen de antigüedad de 10 años. Resultados y conclusiones. La perspectiva de género se convierte en un enfoque necesario para la intervención del fenómeno de la
\end{abstract}

\footnotetext{
*El presente texto se deriva de las reflexiones verbales y escriturales de las estudiantes del pregrado de Psicología de la Universidad Católica Luis Amigó en el marco de su curso complementario "Intervenciones en dinámicas familiares" y de la propuesta de trabajo de grado del profesor Mg. Alexander Rodríguez Bustamante titulado Comunicación familiar desde la terapia familiar sistémica en el marco de la Especialización en Terapia Familiar de la misma Universidad y su Escuela de Posgrados.

** Universidad Católica Luis Amigó. Medellín, Colombia. E-mail: sara.correaag@amigo.edu.co.

(iD orcid.org/0000-0002-1971-6258. Google Scholar

*** Universidad Católica Luis Amigó. Medellín, Colombia. E-mail: yesica.boteroar@amigo.edu.co.

(D) orcid.org/0000-0003-0516-3370. Google Scholar

Universidad Católica Luis Amigó. Medellín, Colombia. E-mail: jenifer.valoyesar@amigo.edu.co.

(iD orcid.org/0000-0003-3999-8827. Google Scholar

*** Universidad Católica Luis Amigó (Escuela de Posgrados). Medellín, Colombia. Universidad Pontificia Bolivariana (Doctorado en Educación). Medellín, Colombia. E-mail: alexander.rodriguezbu@amigo.edu.co, alexander.rodriguez@upb.edu.co. (D) orcid.org/oooo-0001-6478-1414. Google Scholar
}

DOI: 10.17151/rlef.2021.13.2.8. 
violencia filio-parental desde la prevención, en tanto contribuye a la democratización de las funciones y roles en las dinámicas familiares como la afectividad y la autoridad; de tal manera que padres, madres y cuidadores puedan asumir estilos parentales democráticos y de disciplina inductiva en función de la crianza de seres humano más prosociales.

Palabras clave: violencia intrafamiliar, relación padres-hijos, dinámica familiar, perspectiva de género.

\section{Gender perspective in child-parental violence}

Abstract: Child-parental violence is a manifestation of intrafamily violence that is characterized by the violent, intentional and systematic action of children towards their parents or who exercises the role of significant adult. The harms done to victims can include, physical, psychological, emotional and financial dimensions. Objective. To recognize the qualities of the victim and the perpetrator of child-parental violence from a gender perspective. Methodology. This research was carried out under a qualitative approach with a hermeneutic methodology in the state-of-the-art modality. A total of 56 bibliographic references were taken into account privileging articles with a 10-year seniority margin. Results and conclusion. The gender perspective becomes a necessary approach for the intervention of the phenomenon of child-parental violence from prevention as it contributes to the democratization of the roles and functions in family dynamics such as authority and affectivity in such a way that fathers, mothers and caregivers can assume democratic parental styles and inductive discipline based on the upbringing of more prosocial human beings.

Key words: family violence, parent-children relationship, family dynamics, gender perspective. 


\section{Introducción}

"La importancia de los relatos o las narrativas familiares se plantea en términos del papel que cumplen en el desarrollo y mantenimiento relacional de las familias y de sus integrantes"

(Gallego, 2006, p. 152)

La violencia filio-parental es un subtipo de violencia intrafamiliar que, según David y Ramírez (2020), "se refiere a las distintas formas que uno de sus integrantes ejerce violencia sobre otro, como una especie de relación abusiva que se caracteriza dentro de la trama familiar" (p.152). En Colombia, en el análisis de datos del Instituto Nacional de Medicina Legal y Ciencias Forenses (2019), la violencia intrafamiliar presenta 28.645 casos denunciados que se distribuyen en violencia contra el adulto mayor con 2.261 casos, la violencia en contra de niños y adolescentes con 10.794 casos, y la violencia entre otros familiares con 15.590 casos. Dentro de este último tipo de violencia se incluye la violencia filio-parental que corresponde a un 13,26\% de las violencias entre otros familiares, siendo los hijos la tercera población agresora con mayor número de casos para esta distribución de violencia. La violencia filio-parental se define como "el tipo de violencia en donde un hijo presenta comportamientos negativos (físicos y/o mentales) directamente a sus progenitores (o contra aquellos que presenten el rol de progenitores), de forma consciente, intencionada y en repetidas ocasiones" (David y Ramírez, 2020, p. 148).

Este tipo de violencia aparece en dimensiones específicas de maltrato al igual que en la violencia conyugal (Ortega, 2015). Algunas de estas son: la dimensión del maltrato físico que se refiere a todas las manifestaciones de ataque y desvalorización del otro, como golpear, escupir, arañar, empujar o lanzar objetos (Aracoa y Alba, 2012). En segundo lugar se encuentra el maltrato psicológico que abarca las acciones dirigidas a intimidar o atemorizar los progenitores (Aracoa y Alba, 2012). En cuanto al maltrato emocional se relaciona con las mentiras maliciosas, chantajes sobre suicidarse o escapar de casa y realizar exigencias irrealistas (Ortega, 2015). Finalmente, el maltrato financiero incluye el hurto de dinero, destrucción de los bienes materiales de progenitores, y comprar o generar deudas con un valor económico inalcanzable para los cuidadores (Aracoa y Alba, 2012).

\section{Metodología}

El presente artículo de revisión se realizó bajo el enfoque de investigación cualitativa, "caracterizado por ser descriptivo, inductivo, holístico, fenomenológico, estructural-sistémico y ante todo flexible, destaca más la validez que la replicabilidad, trata ante todo de identificar la naturaleza profunda de las realidades y su estructura 
dinámica"(Mesías, 2010, p. 1). Para la interpretación y descubrimiento de los mensajes contenidos dentro de la bibliográfica revisada frente a la realidad objeto de estudio se requirió de una metodología hermenéutica (Arráez et al., 2006). En la modalidad de estado del arte, el cual permite la recolección y análisis documental exhaustivo dentro de un área específica (Molina, 2005).

Las fuentes empleadas con preeminencia para la construcción de este artículo fueron: Revista Latinoamericana de Estudios de Familia, Redalyc.org, Poiésis, Redes y Dialnet. En la búsqueda se privilegió el material bibliográfico con un margen de antigüedad de 10 años y los artículos de revista. Las palabras clave que se tuvieron en cuenta para el rastreo documental fueron: violencia filio-parental, violencia doméstica, violencia intrafamiliar, dinámicas familiares, perspectiva de género, violencia y género. Para la sistematización de la información recolectada fue necesario implementar fichas de contenido textual e interpretativo; y una matriz de división categorial, en la cual se pudieron vislumbrar los contenidos temáticos oportunos para cada apartado del cuerpo del artículo.

Este estudio pretende reconocer las cualidades de la víctima y victimario de la violencia filio-parental en las dinámicas familiares desde una perspectiva de género. Este objetivo se nutre de tres objetivos específicos: en primer lugar, se pretende describir las dinámicas familiares asociadas a la aparición de la violencia filio-parental. El segundo objetivo se centra en describir las características de la víctima de la violencia filio-parental desde una perspectiva de género. Finalmente, el tercer objetivo se dirige a identificar las características del victimario de la violencia filio-parental desde una perspectiva de género.

Las categorías para el objetivo uno se resumieron en afecto, comunicación, autoridad y roles. Para el objetivo dos se abordaron las categorías de perspectiva de género y cualidades de la víctima de la violencia filio parental. Por último, para el objetivo tres, se incluyeron las categorías de perspectiva de género y la categoría de cualidades del victimario de la violencia filio-parental.

\section{Dinámicas familiares y violencia filio-parental}

Según Gallego (2012), las dinámicas familiares son los "encuentros entre las subjetividades, encuentros mediados por una serie de normas, reglas, límites, jerarquías y roles, entre otros, que regulan la convivencia y permite que el funcionamiento de la vida familiar se desarrolle armónicamente" (p. 339). Para efectos de este escrito se diferenciaron algunas características de las dinámicas familiares en la violencia filio-parental. 


\section{Roles}

Según la Real Academia Española (2014), el término rol hace alusión al "papel o función que alguien o algo desempeña" (párr. 1). Dentro del contexto familiar este término tiene el mismo significado y determina la dinámica en cada familia. Gallego (2012) expone que inicialmente los roles familiares tienen relación directa con el género, es decir que el rol está sujeto a las características propias que debe tener la mujer y el hombre, como sentimientos y comportamientos en determinado contexto, cristalizando de esta manera los estereotipos instaurados dentro de la cultura. Por otra parte, los roles también están aunados a los ingresos económicos y cómo estos se relacionan con la distribución de las tareas dentro de la casa. De tal forma que, en sociedades como la colombiana con una tradición patriarcal, el hombre generalmente ha ocupado el lugar de proveedor económico y a la mujer se la ha situado como la persona encargada de los cuidados del hogar, los hijos y el esposo (Gallego, 2012). Según Martínez et al. (2019), "los roles que ejercen los padres de familia se acompañan de la autoridad y la norma, que con la comunicación se correlacionan como elementos fundamentales que forjan herramientas básicas para la socialización” (p. 113).

Es importante destacar que la inserción de la mujer al mercado laboral remunerado ha configurado uno de los factores más influyentes para el inicio de la crisis del patriarcado en los movimientos postmodernos, dando paso a la transición de algunos miembros masculinos de las familias hacia la responsabilización compartida de las tareas domésticas (Montoya-Ahmedt, 2017). Pero, según Gallego (2012), cuando la madre sale del hogar como único contexto de definición de sí misma, quien suple las funciones es la hija mayor, y por su parte el padre y los hijos varones asumen un papel de protectores y cuidadores. Ahora bien, es indefectible mencionar que la transformación de los roles al interior de las unidades familiares puede ser problemática en algunos casos, y para Claver (2017) los roles y funciones difusas y ambivalentes pueden representar un factor de riesgo para el desarrollo de ciclos de violencia filio-parental.

\section{Estilos parentales: comunicación, afecto y autoridad}

Los estilos parentales asumidos en el ejercicio de cuidado de los hijos se constituyen a partir de una relación coyuntural del tipo de comunicación, estilo afectivo y de autoridad del cuidador. Según Ibabe (2015), esta relación se clasifica en cuatro variaciones de estilo parental: (1) autoritario, en donde los cuidadores ejercen altos niveles de control, en contraste con una expresión afectiva precaria; (2) democrático, propio de familias con altos niveles de control y afecto; (3) indulgente, cuando el control está notablemente disminuido en comparación con el afecto; 
Sara Melisa Correa-Agudelo et al.

y (4) negligente, para familias en las que se evidencian bajos niveles de control y afecto. De acuerdo con Ibabe (2015), en el contexto español y latinoamericano:

[...] la falta de afecto e implicación paterna en la educación de los hijos (estilos autoritario y negligente) estarían relacionados con la manifestación de agresividad dirigida hacia los progenitores, mientras que los estilos parentales basados en el afecto (estilos indulgentes y democráticos) se relacionarían con un menor nivel de agresividad verbal $\mathrm{y}$ física de los hijos hacia los padres. (p. 616)

Teniendo en cuenta que estos estilos parentales abarcan el afecto,la comunicación y autoridad, es importante ahondar en estos conceptos. Salazar (2017) sugiere que el afecto es un elemento esencial en relación con la violencia filio-parental, ya que la familia es el grupo primario de socialización del sujeto y las demostraciones afectivas facilitan la creación de vínculos sanos; cuando estas manifestaciones no son claras o se caracterizan por ser rígidas pueden influir en las respuestas comportamentales impulsivas, irritables e incluso agresivas en las dinámicas familiares.

En cuanto a la comunicación, Chunga (2008) menciona que es el proceso que permite la cohesión y adaptabilidad dentro de un sistema familiar. Y cuando esta se define como una comunicación dañada, que para Gallego (2012) se caracteriza por intercambios familiares basados en la sátira, reproche, gritos, fiscalización o imposiciones, se propicia la fractura en las relaciones con los miembros de la familia, por lo que los procesos de autoridad y socialización pueden verse afectados y tornarse en estilos parentales autoritarios o negligentes, que como se mencionó anteriormente pueden ser un factor de riesgo para la aparición de la violencia filio-parental.

\section{Disciplina familiar: autoridad y afecto}

La autoridad se relaciona con el ejercicio de poder y estilos de disciplina de acuerdo con las particularidades de cada sistema familiar. Ibabe (2015) propone tres tipos de disciplina parental en donde se relacionan la autoridad y el estilo de afecto familiar: (1) estrategias coercitivas, en donde comportamientos inadecuados tienen una consecuencia negativa como los castigos; (2) estrategias inductivas, que recurren a la reflexión y negociación con los hijos alrededor de las consecuencias de un mal comportamiento; y (3) las estrategias indiferentes, que se caracterizan por la pasividad y permisividad en los límites para los hijos.

De acuerdo con lo anterior, la disciplina coercitiva se asocia con la aparición de violencia filio-parental. En cuanto a las estrategias menos coercitivas, no hay una relación significativa con la violencia filio-parental (Ibabe, 2015). No obstante, las estrategias con bajos niveles de coerción, especialmente las estrategias indiferentes, 
pueden influir en el deficiente autocontrol de los hijos, la escalada de la violencia y el consumo de sustancias psicoactivas (Ibabe, 2015).

Para Claver (2017), en la medida en que los cuidadores adoptan estilos y estrategias parentales inconsistentes, fluctuando entre la permisividadindulgencia y la coerción-autoritarismo, pueden ser más propensos a sufrir de violencia filio-parental. Según Ortega (2015): "Parece existir una aparente pérdida de autoridad por parte de los padres que ha propiciado un aparente cambio de dirección en torno a la acción violenta” (p. 48). En este sentido, se evidencia que el aumento de la conducta violenta por parte del agresor se debe a que, en la medida en que estos exigen, los padres se muestran más dispuestos a acceder a las peticiones, con el fin de disminuir la tensión en la familia (Molla-Esparza y Aroca-Montolío, 2018). Esto se constituye en un feedback positivo de la acción violenta y aumenta la percepción de los agresores frente a la vulnerabilidad de los ascendentes. Y cuando los cuidadores quieren ejercer un feedback negativo a través de las estrategias coercitivas-autoritarias, el resultado es la escalada reactiva de violencia por parte de los hijos.

\section{Perspectiva de género de la violencia filio-parental}

"Las familias descubren y develan la necesidad de comunicar cualquier situación o evento cotidiano, 'sacarse de sî', describiéndolo con facilidad en un ambiente generador de confianza y favorabilidad en su proceso"

(Rodríguez-Bustamante, 2016, p. 40)

Existen dos líneas de poder notorias en las dinámicas familiares: la primera es la línea de poder generacional que se presenta de padres a hijos; y en segundo lugar está la línea de poder de género, que culturalmente es ejercida de hombres a mujeres (Calveiro citada en Puyana, 2015). En el fenómeno de la violencia filio-parental, la línea de poder asociada a la generación se ha invertido, y los hijos son quienes ejercen el poder desde la agresión como se mencionó anteriormente. En cuanto a la línea de poder de género en este fenómeno, existe un quiebre frente a la inferiorización y subordinación de las mujeres a los varones que enmarca un nuevo punto de partida frente al lugar de los estudios de la mujer, luego estudios de género (Giberti, 2005). Por lo que es imperativo hacer un análisis desde la perspectiva de género que, según Caicedo (2005), es una herramienta conceptual, metodológica y política, que permite vislumbrar relaciones de poder y jerarquías para la comprensión de las dinámicas familiares. 


\section{De la víctima}

Teniendo en cuenta que la violencia filio-parental se enmarca en la violencia intrafamiliar, es menester anotar cifras importantes suministradas por la Estadística Delictiva de la Policía Nacional de Colombia (2020) con fecha de corte del 31 de diciembre de 2020, y con 113.567 casos registrados, de los cuales un 78,1\% conciernen a víctimas de género femenino. Las mujeres adultas representaron 80.325 casos, las adolescentes 2.572, a las menores de 18 años se les atribuye 3.264 casos, y respecto a mujeres sin especificación de grupo de edad se reportan 1.642 casos (Policía Nacional de Colombia, 2020). Un 22,6\% del total de casos registrados atañen a víctimas de género masculino, con 20.144 casos de hombres adultos, 1.157 adolescentes, 3.582 menores de 18 años; 873 casos de hombres sin especificación de grupo de edad, y 8 casos de personas de las que no se reporta género o grupo de edad (Policía Nacional de Colombia, 2020).

Respecto a la estadística para el mismo delito, del 1 de enero de 2021 al 28 de febrero del mismo año, se reportaron 14.737 casos: un 76,6\% responde a víctimas de género femenino, con una representación de 10.740 casos de mujeres adultas, 311 de adolescentes y 251 de mujeres menores de 18 años (Policía Nacional de Colombia, 2021). Un $23,3 \%$ concuerda con víctimas del género masculino, con 3.046 casos correspondientes a hombres adultos, 127 adolescentes y 262 menores de 18 años (Policía Nacional de Colombia, 2021).

Según el Instituto Nacional de Medicina Legal y Ciencias Forenses (2019): "en la violencia entre otros familiares, sigue siendo marcada la diferencia entre sexos; mientras que los hombres fueron víctimas en el 35,41\% de los casos, las mujeres lo fueron en el 64,59\%" (p. 170). Siguiendo los datos anteriores, se puede sugerir que, desde la perspectiva de género, la violencia filio-parental se constituye en una forma de violencia basada en género contra las mujeres, y esta última, para BarragánGamba (2015).

\footnotetext{
Se manifiesta de diferentes maneras y tiene lugar en múltiples espacios, pero posee una raíz única: las asimetrías en las relaciones de poder de los géneros y, por consiguiente, la desigualdad y discriminación universal que sufren las mujeres en lo social, económico, cultural y político. (p. 86)
}

En este sentido, "las familias monoparentales y aquellas donde la madre es el progenitor más débil, es donde existe un mayor riesgo de aparición de este tipo de conducta violenta y desnaturalizada contra los ascendientes" (Aroca, 2012, p. 243). Esto podría explicarse debido a que frecuentemente la madre ocupa el lugar de cuidadora principal, y por lo tanto es la figura con la que hay más posibilidades de enfrentamiento en el ciclo vital de la adolescencia (Ortega, 2015). Es importante aclarar que cuando se hace referencia a una figura débil, se refiere a la percepción 
que el agresor (hijo) tiene frente a la posición de poder de la progenitora; ya que, desde la construcción política y sociohistórica del patriarcado, el hombre ocupa el rol de control y superioridad en instituciones sociales, entre las cuales se incluye la familia (Puyana, 2015). Por lo tanto, la figura del padre es menos propensa a relacionarse con la figura de la víctima en el fenómeno de violencia filio-parental.

Asimismo, los antecedentes asociados a la exposición constante a violencia intrafamiliar, especialmente aquella ejercida por parte del padre hacia la madre, representan un factor de riesgo para la figura materna en la violencia filio-parental (Gámez y Calvete, 2012); debido a que en el ciclo de violencia las conductas de agresión son aprendidas en el contexto socializador de la familia a través de la transmisión intergeneracional (Gámez y Calvete, 2012). Y replicadas hacia la figura percibida como más débil.

Por otro parte, hay que considerar la triple jornada laboral a la que muchas mujeres están sujetas en su cotidianidad, especialmente si son la cabeza de hogares monoparentales. Se entiende por triple jornada de trabajo a las labores cotidianas que actualmente desempeñan las mujeres, las cuales incluyen el trabajo asalariado o remunerado, el cuidado y atención de la casa y los hijos, también llamado trabajo doméstico, y finalmente el trabajo institucional, que se refiere a actividades como matricular a los hijos en el sistema educativo, asistir a las reuniones de padres de familia ${ }^{1}$, ayudar con las tareas asignadas a los hijos desde casa, entre otras actividades (Arpini et al., 2012). En estos casos, "la figura de la madre recordemos, se ve doblemente afectada, ya que la dificultad de conciliar tareas laborales con el tiempo y cuidado de los hijos puede proporcionar una supervisión parental ineficaz en el desarrollo evolutivo de los mismos" (Ortega, 2015, p. 58). Tales estilos de supervisión ineficaz pueden traducirse en estrategias de autoridad coercitivas o indiferentes, $y$ estilos parentales autoritarios y negligentes, características asociadas a las dinámicas familiares que se constituyen en factores de riesgo para la aparición de la violencia de tipo filio-parental.

\section{Del victimario}

En cuanto a la figura del victimario, se puede decir que los hijos varones tienden a ejercer esta violencia con ambos progenitores, en comparación de las hijas que tienden a la violencia de tipo verbal y psicológica dirigida a la figura materna (Jiménez, 2017). La etapa del ciclo vital en la que se ubica la mayor parte de los victimarios es la adolescencia, un "periodo de transición entre la niñez y la vida adulta durante el cual acontece la maduración sexual, empieza el pensamiento de operaciones formales y ocurre la preparación para ingresar al mundo adulto" (Rice, 1997, p. 7).

${ }^{1}$ La cursiva es de los autores, para señalar que la misma expresión excluye a la madre del lugar de poder en la crianza. 
Dentro de los cambios más significativos en esta transición está la relación con la norma, ya que se encuentra en el nivel preconvencional del desarrollo del razonamiento moral, en el cual los sujetos se adaptan a las normas sociales en la medida en que perciban el castigo y deseen evitarlo (Navas, 2009).

Algunos autores como Gallagher (2004), Aracoa y Alba (2012), Gámez y Calvete (2012) y Jiménez (2017) atribuyen la mayor proporción de ataques a los hijos de género masculino según sus hallazgos de investigación. Esto puede responder, en primer lugar, a que logran igualar en la adolescencia en tamaño y fuerza a los cuidadores; y en segundo lugar, los hombres son más propensos a identificarse con padres agresivos y pueden verse más afectados en la separación de los progenitores (Gallagher, 2004). Frente al divorcio es importante aclarar que:

\begin{abstract}
[...] las consecuencias negativas de las separaciones conyugales en los hijos están ligadas a un proceso conflictivo, de modo que estos se ven obligados a tomar decisiones importantes que, por lo general, no están en condiciones de tomar, como por ejemplo elegir a uno de sus padres; esta situación los pone en una posición en el que la culpa, la tristeza, el miedo, la angustia y la ansiedad los envuelve significativamente, y puede llevarlos a un estado de agresividad que naturalizan y que comienza a ser parte de sus relaciones cotidianas, afectando sus vínculos y la capacidad de relacionarse. (Gómez et al., 2020, p. 118)
\end{abstract}

Culturalmente, la figura que se aleja del entorno familiar es la del padre — con la que el agresor de género masculino se identifica- (Gallagher, 2004). Y el cuidado es designado en gran proporción a la madre, desde los procesos legales o extrajudiciales de conciliación en sociedades como la colombiana, y "en muchos casos, los eventos de separación inducen a muchas mujeres a participar en el mercado laboral con los problemas de inserción precaria y sus consecuencias en el conflicto de tiempo para la economía del cuidado" (Pineda, 2010, p. 74). Estos eventos pueden tener consecuencias directas en los estilos parentales o de disciplina familiar identificados como factores de riesgo para la violencia filio-parental, tal y como se mencionó anteriormente.

En cuanto al contexto colombiano, es importante acotar que las fuentes de información estadística respecto a la violencia filio-parental son escasas. En cuanto a la Estadistica Delictiva, suministrada por la Policía Nacional de Colombia, no se especifica información sobre los agresores en el contexto de violencia intrafamiliar. En el caso del Sistema Integrado de Información sobre Violencias de Género (SIVIGE, 2020), de los 7.501 casos de violencia intrafamiliar registrados por la Fiscalía General de la Nación con corte del 26 de octubre de 2020, el 29,48\% corresponde a agresiones por parte de un familiar diferente a la pareja o expareja, con un 64,51\% de víctimas de género femenino (SIVIGE, 2020). Sin embargo, 
no se especifica sexo o grupo de edad de dichos agresores. De igual forma, a través del SIVIGE (2020) se evidencia que de los 22.141 casos registrados por el SIVIGILA con corte del 15 de septiembre de 2020, un 26,27\% corresponde a agresores que son familiares de la víctima diferentes a parejas o exparejas, y de los 2.177 casos reportados de violencia psicológica un 35,47\% tiene como agresor a un familiar diferente a la pareja o expareja; para ambos casos, el SIVIGILA no reporta singularidades de la población victimaria, como sexo, edad, escolaridad u otras características.

De acuerdo con el Instituto Nacional de Medicina Legal y Ciencias Forenses (2019), en Colombia las cifras registradas sobre los presuntos agresores en la violencia filio-parental son más altas para la figura de la hija, con 1.511 casos registrados hasta en 2019, en comparación con los hijos que registran 557 casos en el mismo año. Lo anterior iría en contravía de los hallazgos de varios estudios europeos y estadounidenses relacionados con la prevalencia de victimarios masculinos en este tipo de violencia intrafamiliar.

Según Agustina y Romero (2013), en los estudios sobre este tema realizados en España hasta el 2005, las adolescentes ocupaban una cifra considerablemente más baja en conductas delictivas. No obstante, en lo que se refiere a la cifra de la violencia de tipo filio-parental, el porcentaje de agresiones con una figura victimaria femenina estaba en aumento. Las posturas de los estudios al respecto de los agresores son diversas, y en muchas ocasiones contradictorias, si bien algunas investigaciones recientes sitúan a los hijos como los agresores más prevalentes, en otras se hace la claridad de que las hijas no son las agresoras más prevalentes, pero sí más frecuentes (Aracoa y Alba, 2012). Esta última explicación podría adaptarse al panorama colombiano.

\section{Discusión y resultados}

Diálogos y lenguajes en momentos de exclusión. Volver sobre la pregunta referida a las perspectivas de género y el fenómeno de lo violento implica el cruce multivocálico de quienes han intervenido el fenómeno en el vínculo filio parental. El siguiente recuadro (Tabla 1) potencia y alienta la reflexión en términos de intervención que, a juicio de los autores del presente artículo, resulta y aparece como un reto pendiente y en vía de instauración no solo para aquellos que se encuentran en la primera línea de atención, sino también de las instituciones y profesionales que ejercen en su ejercicio psicosocial alfabetización en promoción y prevención. 
Sara Melisa Correa-Agudelo et al.

Tabla 1. Tamiz referencial con una mirada desde lo familiar

\begin{tabular}{|c|c|c|}
\hline $\begin{array}{l}\text { 1. Fundantes } \\
\text { temáticos }\end{array}$ & $\begin{array}{l}\text { 2. Autoría: encuentros y } \\
\text { desencuentros }\end{array}$ & 3. Voces: sobre lo vincular y relacional \\
\hline $\begin{array}{l}\text { Retos de la } \\
\text { investigación en } \\
\text { familia en clave de } \\
\text { masculinidades }\end{array}$ & $\begin{array}{l}\text { Gallego-Montes (2018). } \\
\text { Palabras clave: } \\
\text { masculinidades, estudios } \\
\text { de familia, paternidad, } \\
\text { trabajo doméstico, violencia } \\
\text { intrafamiliar, Colombia. }\end{array}$ & $\begin{array}{l}\text { "Un elemento importante del cual hay } \\
\text { poco desarrollo investigativo en el país está } \\
\text { relacionado con los varones y la violencia } \\
\text { intrafamiliar. Existe trabajo de intervención } \\
\text { con hombres a partir de la problemática que } \\
\text { se acumula año tras año, pero el ejercicio } \\
\text { reflexivo y comprensivo académico es aún } \\
\text { insuficiente" (p. 46) }\end{array}$ \\
\hline $\begin{array}{l}\text { Construcción en } \\
\text { contexto de género }\end{array}$ & $\begin{array}{l}\text { Lozano-Beltrán (2018). } \\
\text { Palabras clave: transexualidad, } \\
\text { transgénero, transformación, } \\
\text { cuidado, salud. }\end{array}$ & $\begin{array}{l}\text { "Durante el análisis de la información se } \\
\text { detectó gran cantidad de información } \\
\text { relacionada con la violencia por prejuicio de } \\
\text { género, razón por la cual se decidió realizar } \\
\text { un tercer capítulo sobre el tema de violencia } \\
\text { y discriminación específicamente, y las } \\
\text { prácticas de autocuidado desprendidas para } \\
\text { enfrentarlo" (p. 108) }\end{array}$ \\
\hline $\begin{array}{l}\text { La violencia filio- } \\
\text { parental, un asunto } \\
\text { que acentúa otras } \\
\text { violencias }\end{array}$ & $\begin{array}{l}\text { Ubieto (2018). } \\
\text { Palabras clave: } \\
\text { violencia sexual, violencia de } \\
\text { género, estereotipos de género, } \\
\text { consentimiento, victimización } \\
\text { secundaria, control de } \\
\text { convencionalidad. }\end{array}$ & $\begin{array}{l}\text { "Este es un ejemplo muy ilustrativo de } \\
\text { que un cambio en la mentalidad y las } \\
\text { prácticas institucionales — que también } \\
\text { está lejos de alcanzarse- no basta para } \\
\text { erradicar la violencia de género en todas sus } \\
\text { manifestaciones, especialmente cuando los } \\
\text { estereotipos de género y la discriminación se } \\
\text { encuentran tan enraizados en las propias leyes } \\
\text { o normas jurídicas (Douglas, 2012)" (p. 166) }\end{array}$ \\
\hline $\begin{array}{l}\text { Entre lo real y la idea } \\
\text { del acto violento que } \\
\text { no se olvida }\end{array}$ & $\begin{array}{l}\text { Cardoso (2019). } \\
\text { Palabras clave: } \\
\text { violencia de género, violencia en } \\
\text { la pareja, discriminación, sistema } \\
\text { de justicia, Comité de la CEDAW. }\end{array}$ & $\begin{array}{l}\text { "La labor del Comité está en consonancia con } \\
\text { el trabajo de muchas autoras que defienden } \\
\text { que, para erradicar la violencia contra las } \\
\text { mujeres es necesario combatir tanto las raíces } \\
\text { de la desigualdad como los estereotipos } \\
\text { de género que causan y justifican la } \\
\text { discriminación y la violencia (Schneider et al., } \\
2002 \text {, pp. 322, 359; Schneider, 2010, pp. 40- } \\
\text { 42; Maqueda Abreu, 2006, pp. 2-13; Edwards, } \\
2008, \text { pp. 47-58)" (p. 36) }\end{array}$ \\
\hline
\end{tabular}




\begin{tabular}{|c|c|c|}
\hline $\begin{array}{l}\text { El doble significado } \\
\text { desde lo jurídico }\end{array}$ & $\begin{array}{l}\text { Williams (2020). } \\
\text { Palabras clave: } \\
\text { consentimiento, violencia } \\
\text { sexual, abuso sexual, violencia } \\
\text { de género. }\end{array}$ & $\begin{array}{l}\text { "Por un lado, las citadas carencias en el plano } \\
\text { jurídico justifican la necesidad de cambios } \\
\text { en la legislación como mecanismo para } \\
\text { transformar actitudes que normalmente } \\
\text { se han visto influenciados por estereotipos } \\
\text { sexistas y malinterpretaciones sobre la } \\
\text { violencia sexual. Estos prejuicios han estado } \\
\text { presentes en las diferentes figuras jurídicas } \\
\text { relacionadas con esta materia" (p. 153) }\end{array}$ \\
\hline $\begin{array}{l}\text { Violencia filio- } \\
\text { parental: principales } \\
\text { características, } \\
\text { factores de riesgo } \\
\text { y claves para la } \\
\text { intervención }\end{array}$ & $\begin{array}{l}\text { Martínez et al. (2015). } \\
\text { Palabras clave: } \\
\text { child-parent violence, } \\
\text { prevalence, adolescence, risk } \\
\text { factors, intervention. }\end{array}$ & $\begin{array}{l}\text { "Child-parent Violence (hereinafter CPV) } \\
\text { is an increasingly evident problem in } \\
\text { social, health, and judicial protection } \\
\text { systems which, however, continue to } \\
\text { show a number of major deficiencies with } \\
\text { respect to the main characteristics of } \\
\text { CPV, the people involved, the underlying } \\
\text { factors, and efficacious interventions. } \\
\text { Nevertheless, there is a consensus regarding } \\
\text { its devastating consequences. The present } \\
\text { bibliographical review is focused on } \\
\text { analysing the problem of CPV with the aim } \\
\text { of offering useful data for future research } \\
\text { and intervention proposals" (p. 216) }\end{array}$ \\
\hline $\begin{array}{l}\text { Concepciones } \\
\text { sobre la violencia } \\
\text { filio-parental en } \\
\text { el contexto de } \\
\text { Servicios Sociales: un } \\
\text { estudio exploratorio }\end{array}$ & $\begin{array}{l}\text { Moral et al. (2015). } \\
\text { Palabras clave: } \\
\text { violencia filio-parental, familia, } \\
\text { adolescencia, Servicios Sociales, } \\
\text { investigación cualitativa. }\end{array}$ & $\begin{array}{l}\text { "Para finalizar, es importante destacar la } \\
\text { relevancia de esta investigación para la } \\
\text { mejora diaria del trabajo de los profesionales } \\
\text { vinculados a Servicios Sociales, puesto que } \\
\text { la atención a familias que sufren VFP es } \\
\text { una nueva demanda en auge que exige al } \\
\text { profesional el conocimiento de la dinámica } \\
\text { familiar y las características específicas de esta } \\
\text { reciente forma de sufrimiento social" (p. 19) }\end{array}$ \\
\hline
\end{tabular}

Fuente: elaboración propia.

Se inicia el siguiente apartado, denominado Discusión y resultados, con el panóptico anterior, lo cual resulta un inicio para continuar la reflexión. Metodológicamente se nominó el siguiente apartado en un orden temático, el cual se convierte en bisel estructural en la articulación del escrito como Hermeneusis sobre lo filio-parental, y finalmente las Conclusiones. 


\section{Hermeneusis sobre lo filio-parental}

En el análisis hermenéutico del panorama de la violencia filio-parental desde la perspectiva legal y teórica en Colombia, se ha evidenciado que Europa y Estados Unidos de América siguen siendo el referente más cercano para el avance conceptual y legal de este fenómeno en construcción (Instituto Nacional de Medicina Legal y Ciencias Forenses, 2019). En el contexto colombiano hay un importante camino por recorrer partiendo del mismo concepto de familia consignado en la Ley 294 de 1996, que en su artículo tercero define a la familia como la institución básica de la sociedad (Congreso de Colombia, 1996, párr. 9). En la pluralidad de la argumentación la violencia no puede reducirse a un asunto privado ni individual, dado que en sí mismo guarda conexiones potentes en las estructuras estatales y de la misma sociedad en su mundo vincular (Palacio, 2004). Y esta definición de familia es una legitimación de lo que Puyana (2015) considera familismo. Este concepto describe la estereotipación de la tipología de la familia hetero-patriarcal, monogámica y nuclear, desconociendo otras formas familiares. Asimismo, implica la sobrecarga de la familia en funciones y la reducción de la responsabilidad del Estado y la sociedad (Puyana, 2015). Dicha estereotipación y sobrecarga de tareas, incrementa la sensación de culpa y vergüenza en las unidades familiares ante las problemáticas emergentes. Lo cual, explica por qué la violencia filio-parental, siendo un fenómeno que se ha estudiado progresivamente desde los años 80 a nivel mundial, apenas "se encuentra en pleno despertar referente a las denuncias en las entidades judiciales" (David y Ramírez, 2020).

Es menester aclarar que la perspectiva de género de la violencia filio-parental no implica obviar los registros de los padres víctimas y las hijas victimarias, sino que representa una herramienta para vislumbrar las relaciones de poder en las unidades familiares de acuerdo con los contextos sociohistóricos y culturales con sus respectivas transformaciones. Desde este estudio, reconocemos que "ponerle apellido masculino al ejercicio de la violencia y rostro femenino al papel de víctima es encorsetar, es perpetuar los roles tradicionales, y negar o justificar la violencia femenina equivale a ser su cómplice, a legitimarla" (Trujano et al., 2010, p. 351). En la Encuesta Nacional de Demografía y Salud (Ministerio de Salud y Protección Social, 2015) se ubica a los hombres como las principales víctimas de violencia psicológica en relaciones de pareja con un 74,4\% de los casos registrados hasta el año 2015. Si bien las mujeres siguen ocupando los lugares de mayor vulnerabilidad frente a la violencia intrafamiliar, no se puede ignorar que los hombres afectados por la violencia entre otros familiares dentro de la que se encuentran las cifras de violencia filio-parental, representan una cantidad significativa con 5.519 casos registrados (Instituto Nacional de Medicina Legal y Ciencias Forenses, 2019). Al respecto, Trujano et al. (2010) destacan que: 
Es imprescindible subrayar que la perspectiva de género implica que, precisamente por ser determinadas social e históricamente, las relaciones de inequidad, dominación, discriminación y violencia entre hombres y mujeres pueden y deben cambiar. De ahí la importancia de aceptar la multiplicidad de formas de ser masculinas, como hemos reivindicado el derecho a la pluralidad de formas de ser femeninas. Construir nuevos vínculos a partir de las diferencias abrirá posibilidades de complementación, de búsqueda de alternativas y de relaciones con sentido de equidad entre hombres y mujeres. (p. 352)

En el contexto latinoamericano y específicamente el colombiano, desde el feminismo como corriente política apoyada en la perspectiva de género, se ha instado por la importancia del estudio de las masculinidades a lo largo de la historia, especialmente después de los años 80 donde la inserción de la mujer al mercado laboral remunerado implicó una transformación en las dinámicas familiares. En esta lógica se comienza a gestar la crisis del patriarcado, en donde es indefectible la reflexión de las masculinidades, que se comienzan a mover del mercado laboral e industrialmente productivo como ámbito exclusivo del hombre (Montoya-Ahmedt, 2017). Asimismo, la paternidad ya no es equiparable a la proveeduría económica y alimentaria, y se comienzan a instaurar funciones más cercanas al cuidado y la socialización de los hijos (Gallego-Montes, 2018). No obstante la existencia de una oleada de investigaciones sobre masculinidades de los últimos 30 años, en esta investigación no se logró hallar un antecedente en el que se investigara la violencia filio-parental cuya víctima de interés fuese el padre; convirtiéndose entonces este tema como un reto emergente para los estudios familiares con perspectiva de género.

Es así como la perspectiva de género contempla la deslegitimación de cualquier tipo de violencia o vulneración basada en distribuciones asimétricas del poder en función del género. Esto implica la democratización de las máquinas de verdad y tecnología de inscripción de género que, para Preciado (2009), corresponden a las instituciones y prácticas productoras de la verdad, respectivamente; y en la medida en que se evite que sean captadas por discursos parcializados y hegemónicos se podrán tomar decisiones más plurales. En este sentido, la apertura de las máquinas y tecnologías de producción permite de verdad desmitificar las ficciones políticas de familia, hombre y mujer, que estereotipan la distribución del poder; dando paso a las relaciones familiares equitativas y satisfactorias, en donde se reconozca la importancia de la deconstrucción de pautas relacionales a favor del re-conocimiento del otro.

Schmukler (2013) propone un enfoque de prevención de violencias de género partiendo de la democratización familiar como una herramienta de resignificación colectiva de sentidos asociados a la autoridad y género. De acuerdo con esta autora, dicho enfoque estará dirigido tanto a "funcionarios públicos, promotores de programas de desarrollo social, líderes de organizaciones sociales y comunitarias, educadores y 
académicos" (p. 201). Una propuesta que es digna de ser analizada en el territorio colombiano como una vía posible de intervención de la violencia filio-parental.

En cuanto a la figura del agresor, se puede decir que aún es un tema que requiere de contrastación de resultados cercanos al contexto colombiano, y preferiblemente resultados autóctonos del territorio suramericano; debido a que los estudios más recientes pueden ubicar ciertas características de los agresores, pero las estadísticas en Colombia parecen contrariar dichos hallazgos. Por lo tanto, se hace necesario que en futuras investigaciones se haga una diferenciación en aspectos como la frecuencia de las agresiones de acuerdo al victimario o victimaria, tipos y las motivaciones de las mismas.

\section{Conclusiones}

En la naciente producción nacional sobre el tema de violencia filio-parental, hay importantes retos por superar, entre ellos la transformación de las concepciones familistas en los marcos normativos y sociales que generan vergüenza en las familias afectadas por la violencia intrafamiliar, y entorpece la demanda de atención en situaciones en donde los hijos son los agresores. Y para situar este fenómeno como una problemática de interés, es necesario esforzarse a nivel institucional por la gestión del conocimiento en términos cuantitativos en fuentes oficiales, que se quedan cortas en la pormenorización de las características de los agresores en la violencia intrafamiliar, más allá de las relaciones de pareja. La violencia filio-parental puede interpretarse como un tipo de violencia basada en género contra las mujeres, respaldándose en cifras como las del documento Forensis del Instituto Nacional de Medicina Legal y Ciencias Forenses (2019), pero se requiere de una apuesta desde observatorios nacionales, regionales, departamentales y municipales para la difusión, seguimiento y profundización de un tema del que fata mucho por decir.

Aunado a lo anterior, la panorámica estadística apoyada en la perspectiva de género puede contribuir a problematizar un sistema que le ha costado moverse de una tradición sociohistórica y cultural donde las únicas formas de familia aceptables son aquellas monogámicas, heterosexuales, nucleares y con la adjudicación del poder y control al género masculino; limitando las posibilidades de la mujer en la asunción de roles fuera de la expresión afectiva y en términos equitativos de cargas en las funciones. Ahora bien, el sistema patriarcal y machista también afecta a la población masculina de cara al fenómeno de interés de esta investigación, en la medida en que segrega a los hombres del reconocimiento como posibles víctimas de violencia intrafamiliar, debido a que los estereotipos asignados a la masculinidad tienen que ver con poder y control en las unidades familiares, sociales, políticas y económicas.

Por lo tanto, la perspectiva de género se convierte en un enfoque necesario para la intervención del fenómeno de la violencia filio-parental, en tanto contribuye 
a la democratización de las funciones y roles en las dinámicas familiares como las relacionadas a la afectividad y la autoridad. De tal manera que padres, madres y cuidadores puedan asumir estilos parentales y de disciplina equilibrados en función de la crianza de seres humanos más prosociales, superando las barreras de la distribución inequitativa de poder y características en función de género en un sistema patriarcal. Este objetivo se consigue desde las reflexiones colectivas, que repercuten en prácticas cotidianas y modifican las instituciones culturales desde donde se crean las verdades sociales. En este orden de ideas, la reflexión de la violencia filio-parental desde una perspectiva de género es un necesario en el contexto colombiano, que además implicaría diversificar los esfuerzos en la especificación del fenómeno de una región a otra.

Para esto, es elemental que los estudios de género contrahegemónicos se fortalezcan desde las diferentes áreas del conocimiento que tradicionalmente se han encargado de estudiar a las familias y el género, como lo son la antropología, la psicología, la sociología o la filosofía. Adicionalmente, este discurso debe expandirse a otras áreas de conocimiento poco tradicionales en la investigación del tema, ya que todas las formas de conocimiento o profesionalización se asocian inevitablemente a una función social, que puede rebatir las estructuras sociales dominantes o mantenerlas.

\section{Referencias}

Agustina, J. R. y Romero, F. (2013). Análisis criminológico de la violencia filio-parental. Revista de derecho penal y criminologia, 9(3), 225-266. http://revistas.uned.es/index.php/RDPC/article/ view/24571/19464

Aracoa, C. y Alba, J. (2012). La violencia filio-parental en hijos e hijas adolescentes con rasgos de psicopatía. Criminología y justicia, (3), 25-44.

Arpini, P., Castrogiovanni, N. y Epstein, M. (2012). La Triple Jornada: ser pobre y ser mujer. Margen revista de trabajo social y ciencias sociales, (66), 4-22. http://www.margen.org/suscri/margen66/04_ arpini.pdf

Arráez, M., Calles, J. y Moreno de Tovar, L. (2006). La Hermenéutica: una actividad interpretativa. Sapiens. Revista Universitaria de Investigación, 7(2), 171-181.

Barragán-Gamba, D. (2015). La violencia contra las mujeres es cuestión de género. Criterio Libre Jurídico, 12(2), 79-90. https://doi.org/10.18041/crilibjur.2015.v12n2.24203

Caicedo, C. (2005). Lucha contra la violencia intrafamiliar: perspectivas desde la experiencia colombiana. París, Asociación mundial Escuela Instrumento de Paz, 71-97. http://cifedhop.org/Fr/Publications/ Thematique/thematique13/Caicedo.pdf

Cardoso, E. (2019). Violencia por razón de género en la pareja y estereotipos en el sistema de justicia: lecciones del Comité de la CEDAW. FEMERIS: Revista Multidisciplinar de Estudios de Género, 4(3), 29-52. http://dx.doi.org/10.20318/femeris.2019.4927 
Chunga, L. S. (2008). Niveles de satisfacción familiar y de comunicación entre padres e hijos. Avances en psicologia, 16(1), 109-137. http://www.unife.edu.pe/publicaciones/revistas/psicologia/2008/ sastisfaccionfamiliar.pdf

Claver, E. (2017). Aproximación teórica a la violencia filioparental. Redes, (35). http://redesdigital.com. mx/index.php/redes/article/view/194/144

Congreso de Colombia. (1996). Ley 294 de 1996. Bogotá-Colombia. https://www.icbf.gov.co/cargues/ avance/docs/ley_0294_1996.htm

David, D. y Ramírez, L. (2020). Violencia filio-parental, una reflexión en construcción. Poiésis, O(38). https://doi.org/10.21501/16920945.3559

Gallagher, E. (2004). Youth who victimise their parents. Australian and New Zealand Journal of Family Therapy, 25(2), 94-105.

Gallego, A. (2012). Recuperación crítica de los conceptos de familia, dinámica familiar y sus características. Revista Virtual Universidad Católica del Norte, 1(35), 326-345. https://revistavirtual. ucn.edu.co/index.php/RevistaUCN/article/view/364/679

Gallego-Montes, G. (2018). Estudios de familia en clave de masculinidades. Estado de la discusión en Colombia. Revista Latinoamericana de Estudios de Familia, 10(2), 30-50. http://190.15.17.25/ revlatinofamilia/downloads/Rlef10(2)_3.pdf

Gallego, S. (2006). Comunicación familiar: un mundo de construcciones simbólicas y relacionales. Editorial Universidad de Caldas.

Gámez, M. y Calvete, E. (2012). Violencia filioparental y su asociación con la exposición a la violencia marital y la agresión de padres a hijos. Psicothema, 24(2), 277-283. https://repositorio.uam.es/ bitstream/handle/10486/666513/violencia_gamez_p_2012.pdf? sequence=1\&isAllowed=y

Giberti, E. (2005). La familia, a pesar de todo. Noveduc.

Gómez, C., Lopera, E. y Rodríguez, A. (2020). Separación conyugal, efectos en la salud mental de los hijos. Poiésis, (38), 107-129. https://www.funlam.edu.co/revistas/index.php/poiesis/article/ view/3557

Ibabe, I. (2015). Predictores familiares de la violencia filio-parental: el papel de la disciplina familiar. Anales de Psicologia/Annals of Psychology, 31(2), 615-625. https://revistas.um.es/analesps/article/ view/analesps.31.2.174701/175701

Instituto Nacional de Medicina Legal y Ciencias Forenses. (2019). Forensis: datos para la vida. Bogotá, Colombia. https://www.medicinalegal.gov.co/documents/20143/386932/Forensis+2018.pdf/ be4816a4-3da3-1ff0-2779-e7b5e3962d60

Jiménez, S. (2017). Madres victimizadas. Análisis jurídico de la violencia filio parental como un tipo de violencia hacia la mujer.Anales de derecho, 35(1),1-33. https://revistas.um.es/analesderecho/article/ view/289231/216471

Lozano-Beltrán, J. A. (2018). Prácticas de autocuidado y apoyo en mujeres transgénero residentes en Bogotá, durante sus procesos de tránsito de género. Revista Latinoamericana de Estudios de Familia, 10(1), 99-119. http://190.15.17.25/revlatinofamilia/downloads/Rlef10_6.pdf

Martínez,D.,Amaya,D.,y Calle,P.A.(2019).Prácticas de crianza y comunicación familiar: una estrategia para la socialización primaria. Poiésis, (36), 111-125. https://doi.org/10.21501/ 16920945.3193 
Martínez, M. 1., Estévez, E., Jiménez, T. I. y Velilla, C. (2015). Violencia filio-parental: principales características, factores de riesgo y claves para la intervención. Papeles del Psicólogo, 36(3), 216-223. https://www.redalyc.org/articulo.oa?id=778/77842122007

Mesías, O. (2010). La investigación cualitativa. Universidad Central de Venezuela, Doctorado en Urbanismo, Seminario de Tesis. https://scholar.google.com.mx/scholar?hl=es\&lr=lang_es\&as_sdt $=0 \% 2 \mathrm{C} 5 \% \mathrm{q}=\mathrm{Mes} \% \mathrm{C} 3 \% \mathrm{AD}$ as$\% 2 \mathrm{C}+\mathrm{O} .+\% 282010 \% 29 .+\mathrm{La}+$ investigaci\%C3\%B3n+cualitativa. $+\mathrm{U}$ niversidad + Central + de + Venezuela\%2C+Doctorado + en + Urbanismo\%2C+Seminario + de + Tesis. $+\mathrm{R}$ ecuperado+en+http $\% 3 \mathrm{~A} \% 2 \mathrm{~F} \% 2 \mathrm{Fbit} .1 \mathrm{y} \% 2 \mathrm{~F} 30 \mathrm{unp} 0 \mathrm{C} . \& \mathrm{btnG}=$

Ministerio de Salud y Protección Social. (2015). Resumen Ejecutivo. Encuesta Nacional de Demografía y Salud (ENDS). Colombia. https://profamilia.org.co/wp-content/uploads/2019/06/ResumenEjecutivo-Encuesta-Nacional-De-Demografia-Y-Salud-ends-2015.pdf

Molina, N. P. (2005). ¿Qué es el estado del arte? Ciencia y Tecnología para la salud Visual y Ocular, (5), 73-75.

Molla-Esparza, C. y Aroca-Montolío, C. (2018). Menores que Maltratan a sus Progenitores: definición Integral y su Ciclo de Violencia. Anuario de Psicología Jurídica, 28(1), 15-21. https://doi. org/10.1016/j.apj.2017.01.001

Montoya-Ahmedt, C.A. (2017). La paternidad tras la ruptura de pareja: transformaciones derivadas de los procesos de separación. Revista Latinoamericana de Estudios de Familia, 9, 131-147. http://revlatinofamilia.ucaldas.edu.co/downloads/Rlef9_9.pdf

Moral, G. Del, Varela, R. M., Suárez, C. y Muaitu, G. (2015). Concepciones sobre la violencia filioparental en el contexto de Servicios Sociales: un estudio exploratorio. Acción Psicológica, 12(1), 11-22. https://dx.doi.org/10.5944/ap.12.1.12247

Navas, A. L. (2009). La educación moral según Lawrence Kohlberg: una utopía realizable. Praxis filosófica, (28), 7-22.

Ortega, D. (2015). La violencia filio-parental. ¿Un subtipo de violencia de género? Una revisión bibliográfica de la figura de la víctima. RES, Revista de Educación Social, (21), 45-63. http://www. eduso.net/res/winarcdoc.php?id=632

Palacio, M. C. (2004). Familia y violencia familiar. De la invisibilización al compromiso político. Un asunto de reflexión sociológica. Universidad de Caldas y la Fundación para el desarrollo integral de la niñez, la juventud y la familia (FESCO).

Pineda, J. (2010). Familia postmoderna popular, masculinidades y economía del cuidado. Revista Latinoamericana de Estudios de Familia, 2, 51-78. http://revlatinofamilia.ucaldas.edu.co/downloads/ Rlef2_3.pdf

Policía Nacional de Colombia. (2020). Estadística Delictiva. https://www.policia.gov.co/grupoinformaci\%C3\%B3n-criminalidad/estadistica-delictiva

Policía Nacional de Colombia. (2021). Estadística Delictiva. https://www.policia.gov.co/grupoinformaci\%C3\%B3n-criminalidad/estadistica-delictiva

Preciado, B. (2009). La invención del género, o el tecnocordero que devora a los lobos. En Conversaciones Feministas, Biopolítica (pp. 15-38). https:/www.bibliotecafragmentada.org/wp-content/ uploads/2019/05/365213634-Preciado-B-La-Invencion-Del-Genero-o-El-Tecnocordero-QueDevora-a-Los-Lobos-1.pdf 
Sara Melisa Correa-Agudelo et al.

Puyana, Y. (2015). El familismo: una crítica desde la perspectiva de género y el feminismo. Familias, cambios y estrategias, 263-278. http://bdigital.unal.edu.co/1363/19/18CAPI17.pdf

Real Academia Española. (2014). Diccionario de la lengua española (23 ed.). https://dle.rae.es/rol

Rice, F. (1997). Desarrollo humano: estudio del ciclo vital (2 ed.). Prentice-Hall Hispanoamericana, Pearson Educación.

Rodríguez-Bustamante, A. (2016). La comunicación familiar. Una lectura desde la terapia familiar sistémica. Revista Latinoamericana de Estudios de Familia, 8, 26-43. http://revlatinofamilia.ucaldas. edu.co/downloads/Rlef8_3.pdf

Salazar, S. (2017). El clima social familiar como predictor de la violencia Filio-Parental: Un enfoque hacia los estilos de crianza de los padres (tesis de pregrado). Universidad Estatal de Milagro. http://repositorio. unemi.edu.ec/bitstream/123456789/3583/2/EL\%20CLIMA\%20SOCIAL\%20FAMILIAR\%20 COMO\%20PREDICTOR\%20DE\%20LA\%20VIOLENCIA\%20FILIO-PARENTAL\%20 UN\%20ENFOQUE\%20HACIA\%20LOS\%20ESTILOS\%20DE\%20CRIANZA\%20DE\%20 LOS\%20PADRES.pdf

Schmukler, B. (2013). Democratización familiar como enfoque de prevención de violencia de género: experiencias en México. Revista Latinoamericana de Estudios de Familia, 5, 199-221. http://revlatinofamilia.ucaldas.edu.co/downloads/Rlef5_11.pdf

SIVIGE - Sistema Integrado de Información de Violencias de Género. (2020). Indicadores de violencia por razones de género, durante la pandemia COVID-19. Ministerio de Salud y Protección Social de Colombia. https://app.powerbi.com/view?r=eyJrIjoiZjg3YjgyMzctMmQ2OS00YWVjLT g1MTMtMGViNTBhNGZmNzAzIiwidCI6ImE2MmQ2YzdiLT1mNTktNDQ 2OS05MzU5LTM1MzcxNDc1OTRiYiIsImMiOjR9

Trujano, P., Martínez, A. E. y Camacho, S. I. (2010). Varones víctimas de violencia doméstica: un estudio exploratorio acerca de su percepción y aceptación. Diversitas: perspectivas en psicología, 6(2), 339-354. http://www.scielo.org.co/pdf/dpp/v6n2/v6n2a10.pdf

Ubieto, A. (2018). La violencia sexual como violencia de género: una perspectiva desde el derecho internacional de los derechos humanos. FEMERIS: Revista Multidisciplinar de Estudios de Género, 3(2), 165-170. http://dx.doi.org/10.20318/femeris.2018.4324

Williams, I. (2020). ¿Por qué tenemos que reevaluar el consentimiento en el contexto de la violencia sexual? FEMERIS: Revista Multidisciplinar de Estudios de Género, 5(1), 152-155. http://dx.doi. org/10.20318/femeris.2020.5161 